\title{
LARGE-TIME ASYMPTOTICS FOR THE DENSITY OF A BRANCHING WIENER PROCESS
}

\author{
PÁL RÉVÉSZ, ${ }^{*}$ Technische Universität Wien \\ JAY ROSEN, ${ }^{* *}$ City University of New York \\ ZHAN SHI, ${ }^{* * *}$ Université Paris VI
}

\begin{abstract}
Given an $\mathbb{R}^{d}$-valued supercritical branching Wiener process, let $\psi(A, T)$ be the number of particles in $A \subset \mathbb{R}^{d}$ at time $T(T=0,1,2, \ldots)$. We provide a complete asymptotic expansion of $\psi(A, T)$ as $T \rightarrow \infty$, generalizing the work of X. Chen.
\end{abstract}

Keywords: Branching Wiener process; distribution of particles

2000 Mathematics Subject Classification: Primary 60F15; 60J80

\section{Introduction}

Consider the following model in $\mathbb{R}^{d}$ (with $d \geq 1$ ).

(i) A particle starts from the origin in $\mathbb{R}^{d}$ and executes a Wiener process $W(t) \in \mathbb{R}^{d}$.

(ii) Upon arriving, at time $t=1$, at the new location $W(1)$, the particle dies.

(iii) At death it is replaced by $Y$ offspring, where

$$
\mathrm{P}\{Y=\ell\}=p_{\ell}, \quad \ell=0,1,2, \ldots,
$$

and

$$
1<\sum_{\ell=0}^{\infty} \ell p_{\ell}=m<\infty, \quad 0<\sum_{\ell=0}^{\infty}(\ell-m)^{2} p_{\ell}=\sigma^{2}<\infty .
$$

(iv) Each of the offspring executes a Wiener process starting from where its ancestor dies, and repeats steps (ii) and (iii). This process continues indefinitely. All Wiener processes and offspring numbers are assumed to be independent of each other.

Let

$$
\lambda(\boldsymbol{x}, t)= \begin{cases}1 & \text { if } \boldsymbol{x} \in \mathbb{R}^{d} \text { is occupied by a particle at time } t \\ 0 & \text { otherwise. }\end{cases}
$$

We write $\psi(A, t)=\sum_{\boldsymbol{x} \in A} \lambda(\boldsymbol{x}, t)$, which stands for the number of particles located at $A \subset \mathbb{R}^{d}$ at time $t$. In particular, $\psi\left(\mathbb{R}^{d}, t\right)$ is the total number of particles alive at time $t$.

\footnotetext{
Received 12 May 2005.

* Postal address: Institut für Statistik und Wahrscheinlichkeitstheorie, Technische Universität Wien, Wiedner Hauptstrasse 8-10/107, A-1040 Vienna, Austria. Email address: revesz@ci.tuwien.ac.at

** Postal address: Department of Mathematics, College of Staten Island, The City University of New York, Staten Island, New York, NY 10314, USA. Email address: jrosen3@earthlink.net Supported, in part, by grants from the NSF and from PSC-CUNY.

*** Postal address: Laboratoire de Probabilités UMR 7599, Université Paris VI, 4 place Jussieu, F-75252 Paris Cedex 05, France. Email address: zhan@proba.jussieu.fr
} 
Since the branching is supercritical, it is well known [1, p. 9] that

$$
N_{0}:=\lim _{T \rightarrow \infty} \frac{\psi\left(\mathbb{R}^{d}, T\right)}{m^{T}}
$$

almost surely (a.s.) exists (and is finite), and that $\mathrm{P}\left\{N_{0}>0\right\}>0$.

The limit properties of $\psi(A, T)$ as $T \rightarrow \infty$ were studied by Chen [3], who proved the following theorem.

Theorem 1.1. (Chen [3].) There exist random variables $N_{1}$ and $N_{2}\left(N_{1}\right.$ being $\mathbb{R}^{d}$-valued) such that, for any Borel set $A \subset \mathbb{R}^{d}$ with $\int_{A}\|\boldsymbol{x}\|^{2} \mathrm{~d} \boldsymbol{x}<\infty$, we have

$$
(2 \pi T)^{d / 2} \frac{\psi(A, T)}{m^{T}}=N_{0} \int_{A} \mathrm{~d} \boldsymbol{x}-\frac{1}{2 T} \int_{A}\left(N_{0}\|\boldsymbol{x}\|^{2}-2 N_{1} \cdot \boldsymbol{x}+N_{2}\right) \mathrm{d} \boldsymbol{x}+o\left(T^{-1}\right)
$$

a.s. when $T \rightarrow \infty$.

This result plays an important role in [6] in the study of the concentration of particles in the branching process. For a related theorem, see [2].

The goal of this paper is to provide a complete asymptotic expansion for $\psi(A, T) / m^{T}$ as $T \rightarrow \infty$. Let us first introduce some notation.

For $\boldsymbol{\alpha}=\left(\alpha_{1}, \ldots, \alpha_{d}\right) \in \mathbb{Z}_{+}^{d}$ and $\boldsymbol{x}=\left(x_{1}, \ldots, x_{d}\right) \in \mathbb{R}^{d}$, we use the notation

$$
|\boldsymbol{\alpha}|=\alpha_{1}+\cdots+\alpha_{d}, \quad \boldsymbol{\alpha} !=\prod_{i=1}^{d} \alpha_{i} !, \quad \boldsymbol{x}^{\boldsymbol{\alpha}}=\prod_{i=1}^{d} x_{i}^{\alpha_{i}}, \quad M_{\boldsymbol{\alpha}}(A)=\int_{A} \boldsymbol{x}^{\boldsymbol{\alpha}} \mathrm{d} \boldsymbol{x} .
$$

Furthermore, for $\boldsymbol{\beta} \in \mathbb{Z}_{+}^{d}$ we will write $\boldsymbol{\beta} \preceq \boldsymbol{\alpha}$ to mean that $\beta_{i} \leq \alpha_{i}$ for all $i$, and if $\boldsymbol{\beta} \preceq \boldsymbol{\alpha}$ we set

$$
\left(\begin{array}{l}
\boldsymbol{\alpha} \\
\boldsymbol{\beta}
\end{array}\right)=\prod_{i=1}^{d}\left(\begin{array}{c}
\alpha_{i} \\
\beta_{i}
\end{array}\right)
$$

The following theorem is the main result of the paper.

Theorem 1.2. There exist random variables $\left(N_{\boldsymbol{\alpha}}, \boldsymbol{\alpha} \in \mathbb{Z}_{+}^{d}\right)$ such that, for any $k \geq 1$ and any bounded Borel set $A \subset \mathbb{R}^{d}$,

$$
\begin{aligned}
(2 \pi T)^{d / 2} & \frac{\psi(A, T)}{m^{T}} \\
& =\sum_{n=0}^{k} \frac{(-T)^{-n}}{2^{n}} \sum_{|\boldsymbol{\alpha}|=n} \frac{1}{\boldsymbol{\alpha} !} \sum_{\boldsymbol{\beta} \leq 2 \boldsymbol{\alpha}}\left(\begin{array}{c}
2 \boldsymbol{\alpha} \\
\boldsymbol{\beta}
\end{array}\right)(-1)^{|\boldsymbol{\beta}|} M_{\boldsymbol{\beta}}(A) N_{2 \boldsymbol{\alpha}-\boldsymbol{\beta}}+o\left(T^{-k}\right) \text { a.s. }
\end{aligned}
$$

when $T \rightarrow \infty$.

Remark 1.1. The random variables $\left(N_{\boldsymbol{\alpha}}, \boldsymbol{\alpha} \in \mathbb{Z}_{+}^{d}\right)$ are described in the proof of Theorem 1.2. They are limits of explicit martingales related to the branching Wiener process.

Although the distributions of the random variables $\left(N_{\alpha}, \boldsymbol{\alpha} \in \mathbb{Z}_{+}^{d}\right)$ are not known, Theorem 1.2 can nevertheless be used to make predictions to any degree of accuracy. To see this, choose an integer $k$ and disjoint sets $\left(A_{\boldsymbol{\alpha}} \subseteq \mathbb{R}^{d},|\boldsymbol{\alpha}| \leq k\right)$. Consider (1.2) for each $A_{\boldsymbol{\alpha}}$ : we have a linear system of equations with the unknowns $N_{2 \alpha-\beta}$. We can solve this system of equations 
if the corresponding determinant is not equal to 0 . It is easy to see that we can choose the sets $A_{\alpha}$ such that the determinant is not 0 , for any $T(T=1,2, \ldots)$. Observe the number of particles of a branching Wiener process that are located in the given sets $\left(A_{\boldsymbol{\alpha}},|\boldsymbol{\alpha}| \leq k\right)$ at time $T_{0}$. Using these observations, we can evaluate the actual values of the random variables $\left(N_{\boldsymbol{\alpha}},|\boldsymbol{\alpha}| \leq k\right)$, with an error term $o\left(T_{0}^{-k}\right)$. Then, with these values, we can use Theorem 1.2 to find the values of the process $(2 \pi T)^{d / 2} \psi(A, T) / m^{T}$ for any $A \subseteq \mathbb{R}^{d}$ and $T \geq T_{0}$, with an error term $o\left(T_{0}^{-k}\right)$.

The proof of Theorem 1.2 is presented in Section 2. In Section 3, we show that if the offspring distribution $Y$ has $p$ moments for some even integer $p$, then the martingales described in Remark 1.1 converge to the random variables $\left(N_{\boldsymbol{\alpha}}, \boldsymbol{\alpha} \in \mathbb{Z}_{+}^{d}\right)$ in $L^{p}$.

\section{The proof}

We start with a preliminary result concerning the transition kernel of the Wiener process. Let

$$
p_{t}^{(d)}(\boldsymbol{x})=\frac{1}{(2 \pi t)^{d / 2}} \exp \left(-\frac{\|\boldsymbol{x}\|^{2}}{2 t}\right) .
$$

Recall the definition of a Hermite polynomial,

$$
\begin{aligned}
H_{n}(x) & =(-1)^{n} \mathrm{e}^{x^{2}} \frac{\mathrm{d}^{n}}{\mathrm{~d} x^{n}}\left(\mathrm{e}^{-x^{2}}\right) \\
& =n ! \sum_{j=0}^{\lfloor n / 2\rfloor} \frac{(-1)^{j}}{j !(n-2 j) !}(2 x)^{n-2 j}, \quad x \in \mathbb{R},
\end{aligned}
$$

and define

$$
H_{n}(x, t)=\sum_{j=0}^{\lfloor n / 2\rfloor} \frac{n !}{j !(n-2 j) !}\left(\frac{-t}{2}\right)^{j} x^{n-2 j}
$$

such that

$$
H_{n}(x, t)=\left(\frac{t}{2}\right)^{n / 2} H_{n}\left(\frac{x}{\sqrt{2 t}}\right), \quad x \in \mathbb{R}, t>0 .
$$

Here, $\lfloor\cdot\rfloor$ denotes the integer-part function.

Lemma 2.1. For any $t, 0<t<T$, and any $x \in \mathbb{R}$,

$$
p_{T-t}^{(1)}(x)=\frac{1}{(2 \pi T)^{1 / 2}} \sum_{n=0}^{\infty} \frac{(-T)^{-n}}{2^{n} n !} H_{2 n}(x, t) .
$$

Proof. We use the following identity (see, for example, [4, p. 75]): for any $a>0$ and $y \in \mathbb{R}$,

$$
\mathrm{e}^{-a^{2} y^{2}}=\sum_{n=0}^{\infty} \frac{(-1)^{n} a^{2 n}}{2^{2 n} n !\left(1+a^{2}\right)^{n+1 / 2}} H_{2 n}(y) .
$$

Taking $y=x / \sqrt{2 t} \in \mathbb{R}$ and $a=\sqrt{t /(T-t)}$ and multiplying both sides by $(2 \pi(T-t))^{-1 / 2}$, we readily obtain (2.1). 
For $\boldsymbol{\alpha}=\left(\alpha_{1}, \ldots, \alpha_{d}\right) \in \mathbb{Z}_{+}^{d}$ and $\boldsymbol{x}=\left(x_{1}, \ldots, x_{d}\right) \in \mathbb{R}^{d}$, we use the notation

$$
H_{\alpha}(\boldsymbol{x}, t)=\prod_{i=1}^{d} H_{\alpha_{i}}\left(x_{i}, t\right)
$$

Lemma 2.2. For any $t, 0<t<T$, and any $(\boldsymbol{x}, \boldsymbol{y}) \in \mathbb{R}^{d} \times \mathbb{R}^{d}$,

$$
p_{T-t}^{(d)}(\boldsymbol{x})=\frac{1}{(2 \pi T)^{d / 2}} \sum_{n=0}^{\infty} \frac{(-T)^{-n}}{2^{n}} \sum_{|\boldsymbol{\alpha}|=n} \frac{1}{\boldsymbol{\alpha} !} H_{2 \boldsymbol{\alpha}}(\boldsymbol{x}, t)
$$

and

$$
p_{T-t}^{(d)}(\boldsymbol{x}-\boldsymbol{y})=\frac{1}{(2 \pi T)^{d / 2}} \sum_{n=0}^{\infty} \frac{(-T)^{-n}}{2^{n}} \sum_{|\boldsymbol{\alpha}|=n} \frac{1}{\boldsymbol{\alpha} !} \sum_{\boldsymbol{\beta} \preceq 2 \boldsymbol{\alpha}}\left(\begin{array}{c}
2 \boldsymbol{\alpha} \\
\boldsymbol{\beta}
\end{array}\right)(-\boldsymbol{x})^{\boldsymbol{\beta}} H_{2 \boldsymbol{\alpha}-\boldsymbol{\beta}}(\boldsymbol{y}, t) .
$$

Proof. Since

$$
p_{t}^{(d)}(\boldsymbol{x})=\prod_{i=1}^{d} p_{t}^{(1)}\left(x_{i}\right)
$$

for $\boldsymbol{x}=\left(x_{1}, \ldots, x_{d}\right) \in \mathbb{R}^{d}$, (2.2) follows from (2.1). To obtain (2.3), we use the fact that

$$
H_{n}(x+y, t)=\sum_{j=0}^{n}\left(\begin{array}{l}
n \\
j
\end{array}\right) x^{n-j} H_{j}(y, t)
$$

To see this, we recall that $[4$, p. 60]

$$
\sum_{n=0}^{\infty} \frac{s^{n}}{n !} H_{n}(x)=\mathrm{e}^{2 s x-s^{2}}, \quad s \in \mathbb{R}
$$

meaning that

$$
\sum_{n=0}^{\infty} \frac{s^{n}}{n !} H_{n}(x, t)=\mathrm{e}^{s x-t s^{2} / 2}
$$

Then

$$
\sum_{n=0}^{\infty} \frac{s^{n}}{n !} H_{n}(x+y, t)=\mathrm{e}^{s(x+y)-t s^{2} / 2}=\mathrm{e}^{s x} \mathrm{e}^{s y-t s^{2} / 2}=\sum_{k=0}^{\infty} \frac{s^{k} x^{k}}{k !} \sum_{j=0}^{\infty} \frac{s^{j}}{j !} H_{j}(y, t),
$$

and by comparing powers of $s^{n}$ we recover (2.4).

We now turn to the study of the branching Wiener process. Clearly, for any $T \geq 1$ and $A \subset \mathbb{R}^{d}$

$$
\mathrm{E}(\psi(A, T) \mid \mathcal{F}(T-1))=m \int_{A} \sum_{\boldsymbol{y} \in \mathbb{R}^{d}} p_{1}^{(d)}(\boldsymbol{y}-\boldsymbol{x}) \lambda(\boldsymbol{y}, T-1) \mathrm{d} \boldsymbol{x}
$$


where, as usual, $\mathcal{F}(t)$ denotes the $\sigma$-algebra induced by the branching process up to time $t$. A simple argument by induction yields that, for all $t, 0<t<T$,

$$
\mathrm{E}(\psi(A, T) \mid \mathcal{F}(t))=m^{T-t} \int_{A} \sum_{\boldsymbol{y} \in \mathbb{R}^{d}} p_{T-t}^{(d)}(\boldsymbol{y}-\boldsymbol{x}) \lambda(\boldsymbol{y}, t) \mathrm{d} \boldsymbol{x} .
$$

It turns out that $\psi(A, T)$ is quite close to its conditional expectation, as is confirmed by the following results.

Assertion 2.1. (Révész [5, Equation (6.16)].) Fix $\gamma \in(0,1)$ and let $t=\left\lfloor T^{\gamma}\right\rfloor$. Let $A \subset \mathbb{R}^{d}$ be a bounded Borel set, and let $\varepsilon>0$. We then have

$$
\frac{\psi(A, T)}{m^{T}}-\frac{1}{m^{t}} \int_{A} \sum_{\boldsymbol{y}} p_{T-t}^{(d)}(\boldsymbol{y}-\boldsymbol{x}) \lambda(\boldsymbol{y}, t) \mathrm{d} \boldsymbol{x}=o\left(m^{-t /(2+\varepsilon)}\right)
$$

almost surely for $T \rightarrow \infty$.

Assertion 2.2. (Révész [5, Equation (6.11)].) There exists a constant $C \equiv C(m, d)>0$ such that, for all $t, 1 \leq t<T$,

$$
\mathrm{E}\left(\sum_{\boldsymbol{y} \in \mathbb{R}^{d}}\{\lambda(\boldsymbol{y}, T)-\mathrm{E}(\lambda(\boldsymbol{y}, T) \mid \mathcal{F}(t))\}^{2}\right) \leq C \frac{m^{2 T-t}}{(T-t)^{d / 2}} .
$$

Lemma 2.3. Let $\varepsilon>0$. Almost surely for all sufficiently large $t$, we have $\lambda(\boldsymbol{y}, t)=0$ whenever $\|\boldsymbol{y}\|>t^{1+\varepsilon}$.

Proof. This follows from the usual estimate for the tail of the Wiener process, the BorelCantelli lemma, and (1.1).

Lemma 2.4. Let $\boldsymbol{\alpha} \in \mathbb{Z}_{+}^{d}$ and let

$$
V_{\boldsymbol{\alpha}}(t)=\sum_{\boldsymbol{y} \in \mathbb{R}^{d}} H_{\boldsymbol{\alpha}}(\boldsymbol{y}, t) \lambda(\boldsymbol{y}, t)
$$

Then $\left(\left(1 / m^{t}\right) V_{\boldsymbol{\alpha}}(t), t \geq 0\right)$ is a martingale and

$$
N_{\alpha}:=\lim _{t \rightarrow \infty} \frac{V_{\alpha}(t)}{m^{t}}
$$

exists and is finite almost surely.

Proof. We start by proving the martingale property. Recall that $\psi\left(\mathbb{R}^{d}, t\right)$ stands for the total number of particles at time $t$. Thus, by numbering these particles and considering them as all starting from time $t=0$ (many of them share common paths, at least partially), we can write

$$
\sum_{\boldsymbol{y}} H_{\boldsymbol{\alpha}}(\boldsymbol{y}, t) \lambda(\boldsymbol{y}, t)=\sum_{i=1}^{\psi\left(\mathbb{R}^{d}, t\right)} H_{\boldsymbol{\alpha}}\left(W^{(i)}(t), t\right),
$$


where $\left(W^{(i)}, i \geq 1\right)$ is a sequence of $\mathbb{R}^{d}$-valued Wiener processes (which are not independent). Conditioning on $\mathcal{F}(t-1)$ and on $\psi\left(\mathbb{R}^{d}, t\right)$, we have

$$
\begin{aligned}
& \mathrm{E}\left(\sum_{i=1}^{\psi\left(\mathbb{R}^{d}, t\right)} H_{\boldsymbol{\alpha}}\left(W^{(i)}(t), t\right) \mid \mathcal{F}(t-1), \psi\left(\mathbb{R}^{d}, t\right)\right) \\
& =\sum_{i=1}^{\psi\left(\mathbb{R}^{d}, t\right)} H_{\boldsymbol{\alpha}}\left(W^{(i)}(t-1), t-1\right) \\
& =\sum_{i=1}^{\psi\left(\mathbb{R}^{d}, t-1\right)} Y_{i, t-1} H_{\boldsymbol{\alpha}}\left(W^{(i)}(t-1), t-1\right),
\end{aligned}
$$

the last identity following from the fact that many particles at time $t$ come from the same ancestor at time $t-1$, with $Y_{i, t-1}$ denoting the number of offspring from the $i$ th particle at time $t-1$.

Integrating on both sides gives

$$
\begin{aligned}
& \mathrm{E}\left(\sum_{i=1}^{\psi\left(\mathbb{R}^{d}, t\right)} H_{\boldsymbol{\alpha}}\left(W^{(i)}(t), t\right) \mid \mathcal{F}(t-1)\right) \\
& =\sum_{i=1}^{\psi\left(\mathbb{R}^{d}, t-1\right)} \mathrm{E}(Y) H_{\boldsymbol{\alpha}}\left(W^{(i)}(t-1), t-1\right) \\
& \quad=m \sum_{i=1}^{\psi\left(\mathbb{R}^{d}, t-1\right)} H_{\boldsymbol{\alpha}}\left(W^{(i)}(t-1), t-1\right),
\end{aligned}
$$

proving that $t \mapsto\left(1 / m^{t}\right) V_{\boldsymbol{\alpha}}(t)$ is a martingale.

We now show that $\left(\left(1 / m^{t}\right) V_{\alpha}(t), t \geq 0\right)$ converges to a finite limit almost surely. With the above notation, we first write

$$
V_{\boldsymbol{\alpha}}(t)=\sum_{l=1}^{\psi\left(\mathbb{R}^{d}, t\right)} H_{\boldsymbol{\alpha}}\left(W^{(l)}(t), t\right)=\sum_{l=1}^{\psi\left(\mathbb{R}^{d}, t-1\right)} \sum_{m=1}^{Y_{l, t-1}} H_{\boldsymbol{\alpha}}\left(W^{(l, m)}(t), t\right),
$$

where $W^{(l, m)}(t)$ is the $m$ th offspring of the $l$ th particle that dies at time $t-1$. Then we can write

$$
\begin{aligned}
V_{\boldsymbol{\alpha}}^{2}(t)= & \sum_{l=1}^{\psi\left(\mathbb{R}^{d}, t-1\right)} \sum_{m=1}^{Y_{l, t-1}} H_{\boldsymbol{\alpha}}^{2}\left(W^{(l, m)}(t), t\right) \\
& +\sum_{l=1}^{\psi\left(\mathbb{R}^{d}, t-1\right)} \sum_{m \neq n, m, n=1}^{Y_{l, t-1}} H_{\boldsymbol{\alpha}}\left(W^{(l, m)}(t), t\right) H_{\boldsymbol{\alpha}}\left(W^{(l, n)}(t), t\right) \\
& +\sum_{i \neq j, i, j=1}^{\psi\left(\mathbb{R}^{d}, t-1\right)} \sum_{m=1}^{Y_{i, t-1}} \sum_{n=1}^{Y_{j, t-1}} H_{\boldsymbol{\alpha}}\left(W^{(i, m)}(t), t\right) H_{\boldsymbol{\alpha}}\left(W^{(j, n)}(t), t\right) .
\end{aligned}
$$


Therefore,

$$
\begin{aligned}
& \mathrm{E}\left(V_{\boldsymbol{\alpha}}^{2}(t) \mid \mathcal{F}(t-1), \psi\left(\mathbb{R}^{d}, t\right)\right) \\
& =\sum_{i=1}^{\psi\left(\mathbb{R}^{d}, t-1\right)} Y_{i, t-1} \mathrm{E}\left(H_{\boldsymbol{\alpha}}^{2}\left(W^{(i, 1)}(t), t\right) \mid \mathcal{F}(t-1)\right) \\
& \quad+\sum_{i=1}^{\psi\left(\mathbb{R}^{d}, t-1\right)}\left(Y_{i, t-1}^{2}-Y_{i, t-1}\right) H_{\boldsymbol{\alpha}}^{2}\left(W^{(i)}(t-1), t-1\right) \\
& \quad+\sum_{i \neq j, i, j=1}^{\psi\left(\mathbb{R}^{d}, t-1\right)} Y_{i, t-1} Y_{j, t-1} H_{\boldsymbol{\alpha}}\left(W^{(i)}(t-1), t-1\right) H_{\boldsymbol{\alpha}}\left(W^{(j)}(t-1), t-1\right) .
\end{aligned}
$$

Thus,

$$
\begin{aligned}
\mathrm{E}\left(V_{\boldsymbol{\alpha}}^{2}(t) \mid \mathcal{F}(t-1)\right) & \\
= & \sum_{i=1}^{\psi\left(\mathbb{R}^{d}, t-1\right)} m \mathrm{E}\left(H_{\boldsymbol{\alpha}}^{2}\left(W^{(i)}(t), t\right) \mid \mathcal{F}(t-1)\right) \\
& +\sum_{i=1}^{\psi\left(\mathbb{R}^{d}, t-1\right)}\left(\sigma^{2}+m^{2}-m\right) H_{\boldsymbol{\alpha}}^{2}\left(W^{(i)}(t-1), t-1\right) \\
& +\sum_{i \neq j, i, j=1}^{i\left(\mathbb{R}^{d}, t-1\right)} m^{2} H_{\boldsymbol{\alpha}}\left(W^{(i)}(t-1), t-1\right) H_{\boldsymbol{\alpha}}\left(W^{(j)}(t-1), t-1\right) \\
= & \sum_{i=1}^{\left.i \mathbb{R}^{d}, t-1\right)}\left[m \mathrm{E}\left(H_{\boldsymbol{\alpha}}^{2}\left(W^{(i)}(t), t\right) \mid \mathcal{F}(t-1)\right)\right. \\
& \\
&
\end{aligned}
$$

Recall that $\mathrm{E}\left(\psi\left(\mathbb{R}^{d}, t-1\right)\right)=m^{t-1}$ (see [1, p. 9]). It is clear from (2.5) that

$$
\mathrm{E}\left(H_{\alpha}^{2}\left(W^{(1)}(t), t\right)\right)=\alpha ! t^{|\alpha|} .
$$

Hence,

$$
\begin{aligned}
\mathrm{E}\left(V_{\boldsymbol{\alpha}}^{2}(t)\right) & =m^{t-1} \boldsymbol{\alpha} !\left(m t^{|\boldsymbol{\alpha}|}+\left(\sigma^{2}-m\right)(t-1)^{|\boldsymbol{\alpha}|}\right)+m^{2} \mathrm{E}\left(V_{\boldsymbol{\alpha}}^{2}(t-1)\right) \\
& =m^{t-1} \boldsymbol{\alpha} !\left(m\left(t^{|\boldsymbol{\alpha}|}-(t-1)^{|\boldsymbol{\alpha}|}\right)+\sigma^{2}(t-1)^{|\boldsymbol{\alpha}|}\right)+m^{2} \mathrm{E}\left(V_{\boldsymbol{\alpha}}^{2}(t-1)\right),
\end{aligned}
$$

whence

$$
0<\mathrm{E}\left(\frac{V_{\alpha}^{2}(t)}{m^{2 t}}-\frac{V_{\alpha}^{2}(t-1)}{m^{2(t-1)}}\right) \leq c \frac{t^{|\boldsymbol{\alpha}|}}{m^{t}},
$$


where $c$ is a finite constant. Hence, using the fact that $V_{\boldsymbol{\alpha}}(t) / m^{t}$ is a martingale, we have

$$
\begin{aligned}
\mathrm{E}\left(\sum_{t=1}^{\infty}\left|\frac{V_{\boldsymbol{\alpha}}(t)}{m^{t}}-\frac{V_{\boldsymbol{\alpha}}(t-1)}{m^{t-1}}\right|\right) & \leq \sum_{t=1}^{\infty}\left\{\mathrm{E}\left(\left(\frac{V_{\boldsymbol{\alpha}}(t)}{m^{t}}-\frac{V_{\boldsymbol{\alpha}}(t-1)}{m^{t-1}}\right)^{2}\right)\right\}^{1 / 2} \\
& =\sum_{t=1}^{\infty}\left\{\mathrm{E}\left(\frac{V_{\boldsymbol{\alpha}}^{2}(t)}{m^{2 t}}-\frac{V_{\boldsymbol{\alpha}}^{2}(t-1)}{m^{2(t-1)}}\right)\right\}^{1 / 2} \\
& \leq c \sum_{t=1}^{\infty} \frac{t^{|\boldsymbol{\alpha}| / 2}}{m^{t / 2}} \\
& <\infty
\end{aligned}
$$

implying that

$$
\sum_{t=1}^{\infty}\left|\frac{V_{\boldsymbol{\alpha}}(t)}{m^{t}}-\frac{V_{\boldsymbol{\alpha}}(t-1)}{m^{t-1}}\right|<\infty \quad \text { a.s. }
$$

This shows that $\left(\left(1 / m^{t}\right) V_{\alpha}(t), t \geq 0\right)$ converges to a finite limit almost surely.

Remark 2.1. Note that from (2.8), by induction on $t$, we have

$$
\mathrm{E}\left(V_{\boldsymbol{\alpha}}^{2}(t)\right)=m^{t-1} \boldsymbol{\alpha} !\left(\sigma^{2} \sum_{j=1}^{t-1} m^{t-j} j^{|\boldsymbol{\alpha}|}+m \sum_{j=1}^{t} m^{t-j}\left(j^{|\boldsymbol{\alpha}|}-(j-1)^{|\boldsymbol{\alpha}|}\right)\right)
$$

and, therefore,

$$
\mathrm{E}\left(N_{\boldsymbol{\alpha}}^{2}\right)=m^{-1} \boldsymbol{\alpha} !\left(\sigma^{2} \sum_{j=1}^{\infty} m^{-j} j^{|\boldsymbol{\alpha}|}+m \sum_{j=1}^{\infty} m^{-j}\left(j^{|\boldsymbol{\alpha}|}-(j-1)^{|\boldsymbol{\alpha}|}\right)\right) .
$$

Lemma 2.5. Let $\boldsymbol{\alpha} \in \mathbb{Z}_{+}^{d}$, and let $V_{\boldsymbol{\alpha}}$ and $N_{\boldsymbol{\alpha}}$ be as in Lemma 2.4. Then, for any $\varepsilon>0$, we have

$$
\frac{V_{\boldsymbol{\alpha}}(t)}{m^{t}}=N_{\boldsymbol{\alpha}}+o\left(m^{-t /(2+\varepsilon)}\right)
$$

almost surely as $t \rightarrow \infty$.

Proof. We claim that

$$
\frac{V_{\boldsymbol{\alpha}}\left(t^{2}\right)}{m^{t^{2}}}=\mathrm{E}\left(\frac{V_{\boldsymbol{\alpha}}\left(t^{2}\right)}{m^{t^{2}}} \mid \mathcal{F}(t)\right)+o\left(m^{-t /(2+2 \varepsilon)}\right) \quad \text { a.s. }
$$

To see this, we first observe that, by Assertion 2.2, Chebyshev's inequality, and the BorelCantelli lemma,

$$
\max _{\boldsymbol{y} \in \mathbb{R}^{d}}\left|\lambda\left(\boldsymbol{y}, t^{2}\right)-\mathrm{E}\left(\lambda\left(\boldsymbol{y}, t^{2}\right) \mid \mathcal{F}(t)\right)\right|=o\left(m^{t^{2}-t /(2+\varepsilon)}\right)
$$

almost surely for $t \rightarrow \infty$. Combining this estimate with (2.6), Lemma 2.3, and the fact that $\sup _{\|\boldsymbol{y}\| \leq t^{2(1+\varepsilon)}} H_{\boldsymbol{\alpha}}\left(\boldsymbol{y}, t^{2}\right) \leq c t^{2(1+\varepsilon)|\boldsymbol{\alpha}|}$, we obtain (2.9). 
Since $\mathrm{E}\left(V_{\boldsymbol{\alpha}}\left(t^{2}\right) / m^{t^{2}} \mid \mathcal{F}(t)\right)=V_{\boldsymbol{\alpha}}(t) / m^{t}$ (by Lemma 2.4), it follows from (2.9) that

$$
\frac{V_{\alpha}\left(t^{2}\right)}{m^{t^{2}}}-\frac{V_{\boldsymbol{\alpha}}(t)}{m^{t}}=o\left(m^{-t /(2+2 \varepsilon)}\right) \quad \text { a.s. }
$$

As a consequence,

$$
N_{\boldsymbol{\alpha}}-\frac{V_{\boldsymbol{\alpha}}(t)}{m^{t}}=\sum_{j=0}^{\infty}\left(\frac{V_{\boldsymbol{\alpha}}\left(t^{2^{j+1}}\right)}{m^{t^{2^{j+1}}}}-\frac{V_{\boldsymbol{\alpha}}\left(t^{2^{j}}\right)}{m^{t^{2^{j}}}}\right)=o\left(m^{-t /(2+2 \varepsilon)}\right) \quad \text { a.s. }
$$

This completes the proof, since $\varepsilon>0$ is arbitrary.

We have now all the ingredients to prove Theorem 1.2.

Proof of Theorem 1.2. Fix $k \geq 1$, fix $\gamma, 0<\gamma<1 / 2(k+1)$, and let $t=\left\lfloor T^{\gamma}\right\rfloor$. Let $\varepsilon>0$ be such that $(1+\varepsilon) \gamma<1 / 2(k+1)$. We will show that

$$
\begin{aligned}
\frac{\psi(A, T)}{m^{T}}= & \frac{1}{(2 \pi T)^{d / 2}} \sum_{n=0}^{k} \frac{(-T)^{-n}}{2^{n}} \sum_{|\boldsymbol{\alpha}|=n} \frac{1}{\boldsymbol{\alpha} !} \sum_{\boldsymbol{\beta} \leq 2 \boldsymbol{\alpha}}\left(\begin{array}{c}
2 \boldsymbol{\alpha} \\
\boldsymbol{\beta}
\end{array}\right)(-1)^{|\boldsymbol{\beta}|} M_{\boldsymbol{\beta}}(A) \frac{V_{2 \boldsymbol{\alpha}-\boldsymbol{\beta}}(t)}{m^{t}} \\
& +o\left(T^{-(k+d / 2)}\right)+O\left(m^{-t /(2+\varepsilon)}\right)
\end{aligned}
$$

almost surely as $T \rightarrow \infty$, where $V_{2 \alpha-\beta}$ is as defined in (2.6). Theorem 1.2 will then follow from Lemma 2.5.

By Assertion 2.1, we have

$$
\frac{\psi(A, T)}{m^{T}}=\frac{1}{m^{t}} \int_{A} \sum_{\boldsymbol{y} \in \mathbb{R}^{d}} p_{T-t}^{(d)}(\boldsymbol{y}-\boldsymbol{x}) \lambda(\boldsymbol{y}, t) \mathrm{d} \boldsymbol{x}+o\left(m^{-t /(2+\varepsilon)}\right)
$$

almost surely as $T \rightarrow \infty$. On the other hand, we can write

$$
(2 \pi T)^{d / 2} p_{T-t}^{(d)}(\boldsymbol{y}-\boldsymbol{x})=\frac{1}{(1-t / T)^{d / 2}} \exp \left(-\frac{\|\boldsymbol{y}-\boldsymbol{x}\|^{2}}{2(T-t)}\right)=f(z, t, \boldsymbol{x}, \boldsymbol{y}),
$$

where $z=1 / T$ and

$$
f(z, t, \boldsymbol{x}, \boldsymbol{y})=\frac{1}{(1-t z)^{d / 2}} \exp \left(-\frac{\|\boldsymbol{y}-\boldsymbol{x}\|^{2} z}{2(1-t z)}\right)
$$

is a $C^{\infty}$ function of $z$ near $z=0$, for $t z \ll 1$. If we expand $f(z, t, \boldsymbol{x}, \boldsymbol{y})$ in a finite Taylor series in $z$ around $z=0$, it is clear that we can bound the order- $(k+1)$ remainder $R_{k+1}(z, t, \boldsymbol{x}, \boldsymbol{y})$ by a polynomial in $\|\boldsymbol{y}-\boldsymbol{x}\|$ of order at most $2(k+1)$.

According to Lemma $2.3, \lambda(\boldsymbol{y}, t)=0$ as long as $\|\boldsymbol{y}\|>T^{(1+\varepsilon) \gamma}$, almost surely for all sufficiently large $T$. Together with (1.1), which implies that the number of points $y$ with $\lambda(\boldsymbol{y}, t) \neq 0$ is bounded by $\mathrm{cm}^{t}$, and the fact that $A$ is bounded, this implies that

$$
\frac{1}{m^{t}} \int_{A} \sum_{\boldsymbol{y} \in \mathbb{R}^{d}} R_{k+1}\left(T^{-1}, t, \boldsymbol{x}, \boldsymbol{y}\right) \lambda(\boldsymbol{y}, t) \mathrm{d} \boldsymbol{x} \leq c T^{2(1+\varepsilon) \gamma(k+1)}=o(T) .
$$

By inspection of Lemma 2.2, the first $k$ terms in the Taylor series for $f(z, t, \boldsymbol{x}, \boldsymbol{y})$ give rise to the first line of (2.10), completing the proofs of that formula and, hence, of Theorem 1.2. 


\section{3. $L^{p}$-convergence}

In this section, we show that if the offspring distribution $Y$ has $p$ moments for some even integer $p$, then $V_{\boldsymbol{\alpha}}(t) / m^{t}$ converges in $L^{p}$.

Introduce the notation

$$
\widetilde{\sum}_{i_{1}, \ldots, i_{j}=1}^{n}:=\sum_{\substack{i_{1}, \ldots, i_{j}=1 \\ i_{l} \neq i_{m} \forall l \neq m}}^{n}
$$

for summation over unrepeated indices. Let $Z_{t}=\psi\left(\mathbb{R}^{d}, t\right), F_{\boldsymbol{\alpha} ; i}(t)=H_{\boldsymbol{\alpha}}\left(W^{(i)}(t), t\right)$, and

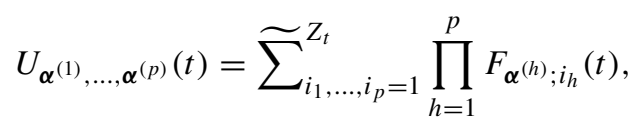

for $\boldsymbol{\alpha}^{(\mathbf{1})}, \ldots, \boldsymbol{\alpha}^{(p)} \in \mathbb{Z}_{+}^{d}$.

The following lemma will play an important role in showing that $V_{\boldsymbol{\alpha}}(t) / m^{t}$ converges in $L^{p}$.

Lemma 3.1. Let $k$ be an integer such that $\mathrm{E}\left(|Y|^{k}\right)<\infty$. Then, for any $\boldsymbol{\alpha}^{(1)}, \ldots, \boldsymbol{\alpha}^{(k)}$, we can find $c, \beta<\infty$ independent of $t$ such that

$$
\left|\mathrm{E}\left(U_{\boldsymbol{\alpha}^{(1)}, \ldots, \boldsymbol{\alpha}^{(k)}}(t)\right)\right| \leq c t^{\beta} m^{k t} .
$$

Proof. We will prove this lemma by induction on $k$. The case $k=1$ is trivial, so assume that the lemma holds for all $k \leq p-1$.

We can write

$$
\begin{aligned}
& U_{\boldsymbol{\alpha}^{(1)}, \ldots, \boldsymbol{\alpha}^{(p)}}(t)={\widetilde{\sum_{i_{1}, \ldots, i_{p}=1}^{Z_{t}}}}_{\prod_{h=1}^{p}} F_{\boldsymbol{\alpha}^{(h)} ; i_{h}}(t) \\
& =\sum_{k=1}^{p} \widetilde{\Sigma}_{i_{1}, \ldots, i_{k}=1}^{Z_{t-1}} \sum_{A_{1} \cup \ldots \cup A_{k}=[1, p]} \prod_{h=1}^{k}\left(\widetilde{\Sigma}_{j_{s}=1 \forall s \in A_{h}}^{Y_{i_{h}, t-1}} \prod_{m \in A_{h}} F_{\boldsymbol{\alpha}^{(m)} ; i_{h}, j_{m}}(t)\right) \text {, }
\end{aligned}
$$

where the sum $\sum_{A_{1} \cup \ldots \cup A_{k}=[1, p]}$ runs over all partitions of $[1, p]=\{1, \ldots, p\}$ into $k$ nonempty sets $A_{1}, \ldots, A_{k}$, and

$$
F_{\boldsymbol{\alpha} ; l, m}(t)=H_{\boldsymbol{\alpha}}\left(W^{(l, m)}(t), t\right) .
$$

Introducing the falling factorial notation $(x)_{k}=x(x-1) \cdots(x-k+1)$, we have

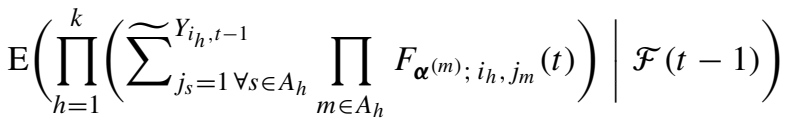

$$
\begin{aligned}
& =\prod_{h=1}^{k} \mathrm{E}\left((Y)_{\left|A_{h}\right|}\right) \prod_{m \in A_{h}} F_{\boldsymbol{\alpha}^{(m)} ; i_{h}}(t-1) .
\end{aligned}
$$


Hence,

$$
\begin{aligned}
& \mathrm{E}\left(U_{\boldsymbol{\alpha}^{(1)}, \ldots, \boldsymbol{\alpha}^{(p)}}(t) \mid \mathcal{F}(t-1)\right) \\
& =\sum_{k=1}^{p} \widetilde{\sum}_{i_{1}, \ldots, i_{k}=1}^{Z_{t-1}} \sum_{A_{1} \cup \ldots \cup A_{k}=[1, p]} \prod_{h=1}^{k} \mathrm{E}\left((Y)_{\left|A_{h}\right|}\right) \prod_{m \in A_{h}} F_{\boldsymbol{\alpha}^{(m)} ; i_{h}}(t-1) \\
& =m^{p} U_{\boldsymbol{\alpha}^{(1)}, \ldots, \boldsymbol{\alpha}}(t)(t-1) \\
& +\sum_{k=1}^{p-1} \widetilde{\Sigma}_{i_{1}, \ldots, i_{k}=1}^{Z_{t-1}} \sum_{A_{1} \cup \ldots \cup A_{k}=[1, p]} \prod_{h=1}^{k} \mathrm{E}\left((Y)_{\left|A_{h}\right|}\right) \prod_{m \in A_{h}} F_{\boldsymbol{\alpha}^{(m)} ; i_{h}}(t-1) .
\end{aligned}
$$

Note that, by (2.5),

$$
\begin{aligned}
\sum_{n=0}^{\infty} \frac{r^{n}}{n !} H_{n}(x, t) \sum_{m=0}^{\infty} \frac{s^{m}}{m !} H_{m}(x, t) & =\mathrm{e}^{r x-t r^{2} / 2} \mathrm{e}^{s x-t s^{2} / 2} \\
& =\exp \left((r+s) x-\frac{t(r+s)^{2}}{2}\right) \mathrm{e}^{t r s} \\
& =\sum_{j=0}^{\infty} \frac{(r+s)^{j}}{j !} H_{j}(x, t) \sum_{k=0}^{\infty} \frac{(t r s)^{k}}{k !} \\
& =\sum_{j=0}^{\infty} \sum_{i=0}^{j} \frac{r^{i} s^{j-i}}{i !(j-i) !} H_{j}(x, t) \sum_{k=0}^{\infty} \frac{(t r s)^{k}}{k !}
\end{aligned}
$$

Equating coefficients of $r^{n} s^{m}$, we find that

$$
H_{n}(x, t) H_{m}(x, t)=n ! m ! \sum_{k=0}^{m \wedge n} \frac{t^{k}}{k !} \frac{1}{(n-k) !(m-k) !} H_{n+m-2 k}(x, t) .
$$

Using this to reduce products of Hermite functions to sums, we find that

$$
\begin{aligned}
\mathrm{E}\left(U_{\boldsymbol{\alpha}^{(1)}, \ldots, \boldsymbol{\alpha}^{(p)}}(t) \mid \mathcal{F}(t-1)\right)= & m^{p} U_{\boldsymbol{\alpha}^{(1)}, \ldots, \boldsymbol{\alpha}^{(p)}}(t-1) \\
& +\sum_{j=1}^{p-1} \sum_{\boldsymbol{\beta}^{(1)}, \ldots, \boldsymbol{\beta}^{(j)}} c\left(\boldsymbol{\alpha} ; p ; \boldsymbol{\beta}^{(1)}, \ldots, \boldsymbol{\beta}^{(j)} ; t\right) U_{\boldsymbol{\beta}^{(1)}, \ldots, \boldsymbol{\beta}^{(j)}}(t-1),
\end{aligned}
$$

where $\sum_{\boldsymbol{\beta}^{(1)}, \ldots, \boldsymbol{\beta}^{(j)}}$ is a finite sum over $\boldsymbol{\beta}^{(1)}, \ldots, \boldsymbol{\beta}^{(j)}$ such that $\sum_{l=1}^{j}\left|\boldsymbol{\beta}^{(l)}\right| \leq \sum_{l=1}^{p}\left|\boldsymbol{\alpha}^{(l)}\right|$, and the $c\left(\boldsymbol{\alpha} ; p ; \boldsymbol{\beta}^{(i)}, \ldots, \boldsymbol{\beta}^{(j)} ; t\right)$ are polynomials in $t$. Hence, by our induction hypothesis,

$$
\mathrm{E}\left(U_{\boldsymbol{\alpha}^{(1)}, \ldots, \boldsymbol{\alpha}^{(p)}}(t)\right)=m^{p} \mathrm{E}\left(U_{\boldsymbol{\alpha}^{(1)}, \ldots, \boldsymbol{\alpha}^{(p)}}(t-1)\right)+\mathcal{R}_{\boldsymbol{\alpha}^{(1)}, \ldots, \boldsymbol{\alpha}^{(p)}}(t),
$$

with $\left|\mathcal{R}_{\boldsymbol{\alpha}^{(1)}, \ldots, \boldsymbol{\alpha}(p)}(t)\right| \leq c t^{\beta} m^{(p-1)(t-1)}$ for some $\beta, c<\infty$ independent of $t$. Iterating this completes the proof of our lemma for $k=p$ and, hence, by induction, for all $k$.

Proposition 3.1. Let $p$ be an even integer such that $\mathrm{E}\left(|Y|^{p}\right)<\infty$. Then $V_{\boldsymbol{\alpha}}(t) / m^{t}$ converges in $L^{p}$. 
Proof. Note that, because of the presence of the polynomial factor $t^{\beta}$ in (3.1), we cannot simply use Lemma 3.1 to show that $V_{\boldsymbol{\alpha}}(t) / m^{t}$ is bounded uniformly in $L^{p}$. Rather, we will show, for some $c, \beta<\infty$ independent of $t$, that

$$
\left|\mathrm{E}\left(\left\{V_{\boldsymbol{\alpha}}(t)-m V_{\boldsymbol{\alpha}}(t-1)\right\}^{p}\right)\right| \leq c t^{\beta} m^{t(p-1)} .
$$

Then

$$
\left|\mathrm{E}\left(\left\{\frac{V_{\boldsymbol{\alpha}}(t)}{m^{t}}-\frac{V_{\boldsymbol{\alpha}}(t-1)}{m^{t-1}}\right\}^{p}\right)\right| \leq c t^{\beta} m^{-t}
$$

and, therefore (it is here that we require $p$ to be even),

$$
\sum_{t=1}^{\infty}\left\|\frac{V_{\boldsymbol{\alpha}}(t)}{m^{t}}-\frac{V_{\boldsymbol{\alpha}}(t-1)}{m^{t-1}}\right\|_{p} \leq c \sum_{t=1}^{\infty} t^{\beta / p} m^{-t / p}<\infty
$$

where $\|\cdot\|_{p}=\left(\mathrm{E}\left(|\cdot|^{p}\right)\right)^{1 / p}$, which will complete the proof of the proposition.

The basic idea of the proof of (3.3) is that the subtraction eliminates the highest-order term in the expectation, leaving only sums of terms of the form $U_{\boldsymbol{\alpha}^{(1)}, \ldots, \boldsymbol{\alpha}^{(k)}}(t), k \leq p-1$. In fact, we have

$$
\begin{aligned}
& \mathrm{E}\left(\left\{V_{\boldsymbol{\alpha}}(t)-m V_{\boldsymbol{\alpha}}(t-1)\right\}^{p}\right) \\
& \quad=\sum_{k=0}^{p}\left(\begin{array}{l}
p \\
k
\end{array}\right)(-1)^{k} m^{k} \mathrm{E}\left(V_{\boldsymbol{\alpha}}^{p-k}(t) V_{\boldsymbol{\alpha}}^{k}(t-1)\right) \\
& \quad=\sum_{k=0}^{p}\left(\begin{array}{l}
p \\
k
\end{array}\right)(-1)^{k} m^{k} \mathrm{E}\left(\mathrm{E}\left(V_{\boldsymbol{\alpha}}^{p-k}(t) \mid \mathcal{F}(t-1)\right) V_{\boldsymbol{\alpha}}^{k}(t-1)\right) .
\end{aligned}
$$

By (2.7), we have

$$
V_{\boldsymbol{\alpha}}(t)=\sum_{l=1}^{Z_{t-1}} \sum_{m=1}^{Y_{l, t-1}} F_{\boldsymbol{\alpha} ; l, m}(t)
$$

Thus,

$$
\begin{aligned}
& V_{\alpha}^{n}(t)=\sum_{j=1}^{n} \widetilde{\Sigma}_{i_{1}, \ldots, i_{j}=1}^{Z_{t-1}} \sum_{l_{1}+\cdots+l_{j}=n}\left(\begin{array}{c}
n \\
l_{1}, \ldots, l_{j}
\end{array}\right) \prod_{h=1}^{j}\left(\sum_{r=1}^{Y_{i_{h}, t-1}} F_{\boldsymbol{\alpha} ; i_{h}, r}(t)\right)^{l_{h}} \\
& =\widetilde{\sum}_{i_{1}, \ldots, i_{n}=1}^{Z_{t-1}} \prod_{h=1}^{n}\left(\sum_{r=1}^{Y_{i_{h}, t-1}} F_{\boldsymbol{\alpha} ; i_{h}, r}(t)\right) \\
& +\sum_{j=1}^{n-1} \widetilde{\sum}_{i_{1}, \ldots, i_{j}=1}^{Z_{t-1}} \sum_{l_{1}+\cdots+l_{j}=n}\left(\begin{array}{c}
n \\
l_{1}, \ldots, l_{j}
\end{array}\right) \prod_{h=1}^{j}\left(\sum_{r=1}^{Y_{i_{h}, t-1}} F_{\boldsymbol{\alpha} ; i_{h}, r}(t)\right)^{l_{h}}
\end{aligned}
$$

and

$$
\left(\sum_{r=1}^{Y_{i_{h}, t-1}} F_{\boldsymbol{\alpha} ; i_{h}, r}(t)\right)^{l_{h}}=\sum_{s=1}^{l_{h}} \widetilde{\sum}_{r_{1}, \ldots, r_{s}=1}^{Y_{i_{h}, t-1}} \sum_{q_{1}+\cdots+q_{s}=l_{h}}\left(\begin{array}{c}
l_{h} \\
q_{1}, \ldots, q_{s}
\end{array}\right) \prod_{f=1}^{s} F_{\boldsymbol{\alpha} ; i_{h}, r_{f}}^{q_{f}}(t),
$$


and we see that

$$
\begin{aligned}
& \mathrm{E}\left(\left(\sum_{r=1}^{Y_{i_{h}, t-1}} F_{\boldsymbol{\alpha} ; i_{h}, r}(t)\right)^{l_{h}} \mid \mathcal{F}(t-1)\right) \\
& \quad=\sum_{s=1}^{l_{h}} \mathrm{E}\left((Y)_{s}\right) \sum_{q_{1}+\cdots+q_{s}=l_{h}}\left(\begin{array}{c}
l_{h} \\
q_{1}, \ldots, q_{s}
\end{array}\right) \prod_{f=1}^{s} \mathrm{E}\left(F_{\alpha ; i_{h}}^{q_{f}}(t) \mid \mathcal{F}(t-1)\right) .
\end{aligned}
$$

Using (3.2) to reduce products of Hermite functions to sums, we find that, by (3.5)-(3.7), we can write

$$
\begin{aligned}
\mathrm{E}\left(V_{\boldsymbol{\alpha}}^{n}(t) \mid \mathcal{F}(t-1)\right)= & m^{n} U_{\boldsymbol{\alpha}^{(1)}, \ldots, \boldsymbol{\alpha}^{(n)}}(t-1) \\
& +\sum_{j=1}^{n-1} \sum_{\boldsymbol{\beta}^{(1)}, \ldots, \boldsymbol{\beta}^{(j)}} c\left(\boldsymbol{\alpha} ; n ; \boldsymbol{\beta}^{(1)}, \ldots, \boldsymbol{\beta}^{(j)} ; t\right) U_{\boldsymbol{\beta}^{(1)}, \ldots, \boldsymbol{\beta}^{(j)}}(t-1),
\end{aligned}
$$

with $\boldsymbol{\alpha}^{(i)}=\boldsymbol{\alpha}, i=1, \ldots, n$, where, again, the $c\left(\boldsymbol{\alpha} ; n ; \boldsymbol{\beta}^{(1)}, \ldots, \boldsymbol{\beta}^{(j)} ; t\right)$ are polynomials in $t$ and $\sum_{\boldsymbol{\beta}^{(1)}, \ldots, \boldsymbol{\beta}^{(j)}}$ is a finite sum.

We next observe that

$$
\begin{aligned}
V_{\boldsymbol{\alpha}}^{n}(t-1)= & \left(\sum_{l=1}^{Z_{t-1}} F_{\boldsymbol{\alpha} ; l}(t)\right)^{n} \\
= & \sum_{j=1}^{n} \widetilde{\Sigma}_{i_{1}, \ldots, i_{j}=1}^{Z_{t-1}} \sum_{l_{1}+\ldots+l_{j}=n}\left(\begin{array}{c}
n \\
l_{1}, \ldots, l_{j}
\end{array}\right) \prod_{h=1}^{j} F_{\boldsymbol{\alpha} ; i_{h}}^{l_{h}}(t-1) \\
= & U_{\boldsymbol{\alpha}^{(1)}, \ldots, \boldsymbol{\alpha}^{(n)}}(t-1) \\
& +\sum_{j=1}^{n-1} \widetilde{\Sigma}_{i_{1}, \ldots, i_{j}=1}^{Z_{t-1}} \sum_{l_{1}+\cdots+l_{j}=n}\left(\begin{array}{c}
n \\
l_{1}, \ldots, l_{j}
\end{array}\right) \prod_{h=1}^{j} F_{\boldsymbol{\alpha} ; i_{h}}^{l_{h}}(t-1) \\
= & U_{\boldsymbol{\alpha}^{(1)}, \ldots, \boldsymbol{\alpha}^{(n)}(t-1)}(t) \\
& +\sum_{j=1}^{n-1} \sum_{\boldsymbol{\gamma}^{(1)}, \ldots, \boldsymbol{\gamma}^{(j)}} d\left(\boldsymbol{\alpha} ; n ; \boldsymbol{\gamma}^{(1)}, \ldots, \boldsymbol{\gamma}^{(j)} ; t\right) U_{\boldsymbol{\gamma}^{(1)}, \ldots, \boldsymbol{\gamma}^{(j)}}(t-1),
\end{aligned}
$$

where we have again used (3.2) to reduce products of Hermite functions to sums, $\sum_{\boldsymbol{\gamma}^{(1)}, \ldots, \boldsymbol{\gamma}^{(j)}}$ is a finite sum, and the $d\left(\boldsymbol{\alpha} ; n ; \boldsymbol{\gamma}^{(1)}, \ldots, \boldsymbol{\gamma}^{(j)} ; t\right)$ are polynomials in $t$.

Similarly,

$$
\begin{aligned}
& U_{\boldsymbol{\beta}^{(1)}, \ldots, \boldsymbol{\beta}^{(j)}}(t-1) U_{\boldsymbol{\gamma}^{(1)}, \ldots, \boldsymbol{\gamma}^{(k)}}(t-1) \\
&=\left(\widetilde{\sum}_{i_{1}, \ldots, i_{j}=1}^{Z_{t-1}} \prod_{h=1}^{j} F_{\boldsymbol{\beta}^{(h)} ; i_{h}}(t-1)\right)\left(\widetilde{\Sigma}_{j_{1}, \ldots, j_{k}=1}^{Z_{t-1}} \prod_{l=1}^{k} F_{\boldsymbol{\gamma}^{(l)} ; j_{l}}(t-1)\right) \\
&= U_{\boldsymbol{\beta}^{(1)}, \ldots, \boldsymbol{\beta}^{(j)}, \boldsymbol{\gamma}^{(1)}, \ldots, \boldsymbol{\gamma}^{(k)}}(t-1) \\
&+\sum_{m=1}^{j+k-1} \sum_{\boldsymbol{\zeta}^{(1)}, \ldots, \boldsymbol{\zeta}^{(m)}} f\left(\boldsymbol{\beta}, \boldsymbol{\gamma} ; \boldsymbol{\zeta}^{(1)}, \ldots, \boldsymbol{\zeta}^{(m)} ; t\right) U_{\boldsymbol{\zeta}^{(1)}, \ldots, \zeta^{(m)}}(t-1),
\end{aligned}
$$


where we have written $\boldsymbol{\beta}=\left(\boldsymbol{\beta}^{(1)}, \ldots, \boldsymbol{\beta}^{(j)}\right)$ and $\boldsymbol{\gamma}=\left(\boldsymbol{\gamma}^{(1)}, \ldots, \boldsymbol{\gamma}^{(k)}\right), \sum_{\zeta^{(1)}, \ldots, \zeta^{(m)}}$ is a finite sum, and the $f\left(\boldsymbol{\beta} ; \boldsymbol{\gamma} ; \boldsymbol{\zeta}^{(1)}, \ldots, \boldsymbol{\zeta}^{(m)} ; t\right)$ are polynomials in $t$.

By combining (3.8)-(3.10) we have, for each $k \leq p$,

$$
\begin{aligned}
& m^{k} \mathrm{E}\left(V_{\boldsymbol{\alpha}}^{p-k}(t) \mid \mathcal{F}(t-1)\right) V_{\boldsymbol{\alpha}}^{k}(t-1) \\
& \quad=m^{p} U_{\boldsymbol{\alpha}^{(1)}, \ldots, \boldsymbol{\alpha}^{(p)}}(t-1) \\
& \quad+\sum_{j=1}^{p-1} \sum_{\boldsymbol{\gamma}^{(1)}, \ldots, \boldsymbol{\gamma}^{(j)}} h\left(\boldsymbol{\alpha} ; n ; \boldsymbol{\gamma}^{(1)}, \ldots, \boldsymbol{\gamma}^{(j)} ; t\right) U_{\boldsymbol{\gamma}^{(1)}, \ldots, \boldsymbol{\gamma}^{(j)}}(t-1),
\end{aligned}
$$

where the $h\left(\boldsymbol{\alpha} ; n ; \boldsymbol{\gamma}^{(1)}, \ldots, \boldsymbol{\gamma}^{(j)} ; t\right)$ are polynomials in $t$ and $\sum_{\boldsymbol{\gamma}^{(1)}, \ldots, \boldsymbol{\gamma}^{(j)}}$ is a finite sum. Substituting this into (3.4) and using the fact that $\sum_{k=0}^{p}\left(\begin{array}{l}p \\ k\end{array}\right)(-1)^{k}=0$, we find that the factors of $m^{p} U_{\boldsymbol{\alpha}^{(1)}, \ldots, \boldsymbol{\alpha}^{(p)}}(t-1)$ cancel, and we can write

$$
\begin{aligned}
& \mathrm{E}\left(\left\{V_{\boldsymbol{\alpha}}(t)-m V_{\boldsymbol{\alpha}}(t-1)\right\}^{p}\right) \\
& \quad=\sum_{j=1}^{p-1} \sum_{\boldsymbol{\gamma}^{(1)}, \ldots, \boldsymbol{\gamma}^{(j)}} g\left(\boldsymbol{\alpha} ; n ; \boldsymbol{\gamma}^{(1)}, \ldots, \boldsymbol{\gamma}^{(j)} ; t\right) \mathrm{E}\left(U_{\boldsymbol{\gamma}^{(1)}, \ldots, \boldsymbol{\gamma}^{(j)}}(t-1)\right),
\end{aligned}
$$

where the $g\left(\boldsymbol{\alpha} ; n ; \boldsymbol{\gamma}^{(1)}, \ldots, \boldsymbol{\gamma}^{(j)} ; t\right)$ are polynomials in $t$. The proofs of (3.3) and, hence, of Proposition 3.1 then follow from (3.1).

Remark 3.1. Note that, by Proposition 3.1, $\left\|V_{\boldsymbol{\alpha}}(t) / m^{t}\right\|_{p}$ is bounded uniformly in $t$, meaning that

$$
\left\|V_{\alpha}(t)\right\|_{p} \leq \mathrm{cm}^{t} .
$$

Arguing as before, any $U_{\boldsymbol{\alpha}^{(1)}, \ldots, \boldsymbol{\alpha}^{(p)}}(t)$ (where $\boldsymbol{\alpha}^{(1)}, \ldots, \boldsymbol{\alpha}^{(k)}$ are now arbitrary) can be written as

$$
U_{\boldsymbol{\alpha}^{(1)}, \ldots, \boldsymbol{\alpha}^{(p)}}(t)=\prod_{i=1}^{p} V_{\boldsymbol{\alpha}^{(i)}}(t)+\text { terms of 'lower order'. }
$$

Thus, using (3.11), Hölder's inequality, and (3.1) for $k \leq p-1$, we can refine (3.1) and find $c, \beta<\infty$ independent of $t$ such that

$$
\left|\mathrm{E}\left(U_{\boldsymbol{\alpha}^{(1)}, \ldots, \boldsymbol{\alpha}}^{(p)}(t)\right)\right| \leq c m^{p t}
$$

(Here we require that $Y$ has $r$ moments for some even $r \geq p$.)

\section{References}

[1] Athreya, K. B. And Ney, P. E. (1972). Branching Processes. Springer, New York.

[2] Biggins, J. D. (1992). Uniform convergence of martingales in the branching random walk. Ann. Prob. 20, 137-151.

[3] Chen, X. (2001). Exact convergence rates for the distribution of particles in branching random walks. Ann. Appl. Prob. 11, 1242-1262.

[4] Lebedev, N. N. (1972). Special Functions and Their Applications. Dover, New York.

[5] RÉvész, P. (1994). Random Walks of Infinitely Many Particles. World Scientific, Singapore.

[6] RÉvész, P. (2004). A prediction problem of the branching random walk. In Stochastic Methods and Their Applications (J. Appl. Prob. Spec. Vol. 41A), Applied Probability Trust, Sheffield, pp. 25-31. 